\title{
Collective intersubband transitions in quantum wells: A comparative density-functional study
}

\author{
C. A. Ullrich and G. Vignale \\ Department of Physics, University of Missouri, Columbia, Missouri 65211
}

(Received 22 June 1998)

\begin{abstract}
We use linearized time-dependent (current) density-functional theory to study collective transitions between the two lowest subbands in $\mathrm{GaAs} / \mathrm{Al}_{x} \mathrm{Ga}_{1-x}$ As quantum wells. We focus on two particular systems, for both of which experimental results are available: a wide single square well and a narrow asymmetric double quantum well. The aim is to calculate the frequency and linewidth of collective electronic modes damped through electron-electron interaction only. Since Landau damping, i.e., creation of single electron-hole pairs, is not effective here, the dominant damping mechanism involves dynamical exchange-correlation effects such as multipair production. To capture these effects, one has to go beyond the widely used adiabatic local-density approximation (ALDA) and include retardation effects. We perform a comparative study of two approaches which fall in this category. The first one is the dynamical extension of the ALDA by Gross and Kohn. The second one is a more recent approach which treats exchange and correlation beyond the ALDA as viscoelastic stresses in the electron liquid. It is found that the former method is more robust: it performs similarly for strongly different degrees of collectivity of the electronic motion. Results for single and double quantum wells compare reasonably to experiment, with a tendency towards overdamping. The viscoelastic approach, on the other hand, is superior for systems where the electron dynamics is predominantly collective, but breaks down if the local velocity field is too rapidly varying, which is the case for single-electron-like behavior such as tunneling through a potential barrier. [S0163-1829(98)01748-2]
\end{abstract}

\section{INTRODUCTION}

In recent years, time-dependent density-functional theory (TDDFT) (Refs. 1 and 2) has become the method of choice for describing dynamical electronic properties of atoms, molecules, and solids in the linear regime and beyond. Until now, TDDFT has been mostly applied within the adiabatic local-density approximation (ALDA) (Ref. 3) for the dynamical exchange-correlation (xc) potential. However, some propositions have been put forward for improving upon the ALDA. The objective of earlier attempts ${ }^{4,5}$ was to obtain approximations for the xc potential which would still be local in space, but not in time. All these approximations were found to suffer from inconsistencies, such as the failure to satisfy the so-called "harmonic potential theorem" (HPT) ${ }^{5-7}$ and other basic symmetries. Only recently has it become clear that the root of these difficulties lies in the fact that the xc potential in TDDFT is an intrinsically nonlocal functional of the density, that is, a functional that does not admit an expansion in terms of the density gradients. ${ }^{8,9}$ Consequently, the most recent extensions of TDDFT beyond the ALDA are formulated in terms of the local current density ${ }^{10}$ or the motion of local fluid elements of the electron liquid. ${ }^{11}$

Among the variety of phenomena which have been investigated using TDDFT methods (see Ref. 2 for a recent review), collective electronic excitations in semiconductor heterostructures are currently of great theoretical and experimental interest. In particular, much effort has gone into the study of parabolic quantum wells with and without imperfections. ${ }^{12-16}$ However, the only collective mode that a uniform external field can excite in a parabolic well is the well-known center-of-mass mode, ${ }^{7}$ which involves no internal compression of the electron gas. We therefore focus in this paper on the electronic response in single ${ }^{17,18}$ and double ${ }^{19-23}$ square wells where no such restriction exists.

The need for going beyond the ALDA becomes evident if one is interested in the linewidth of these electronic excitations. The ALDA accounts for Landau damping of collective modes, i.e., decay into single particle-hole pairs. ${ }^{24}$ For highfrequency, long-wavelength modes, however, this decay mechanism is not effective, and damping is instead induced by dynamical xc effects such as multipair production. In other words, outside the regime where Landau damping occurs, the modes calculated within the ALDA will come out undamped. This limitation is overcome in nonadiabatic theories. In particular, in the formalism of Vignale, Ullrich, and Conti (VUC), ${ }^{10}$ dynamical exchange and correlation lead to the appearance of viscoelastic stresses in the electron fluid, with complex and frequency-dependent viscosity coefficients depending on properties of the homogeneous electron gas. The viscosity causes an additional damping not contained in the ALDA.

In an earlier paper, ${ }^{25}$ the VUC formalism was used in a model system of two-dimensional quantum strips, where it predicts a substantial inhomogeneity-induced enhancement of the damping of collective modes compared to plasmons in the two-dimensional electron gas. The interest in this model system is based on two reasons: first of all, it is analytically solvable, and second, it satisfies the conditions of validity of the approach, namely $\left|\nabla n_{0}(\mathbf{r})\right| / n_{0}(\mathbf{r}) \ll k_{F}(\mathbf{r})$ and $\omega / v_{F}(\mathbf{r})$, where $n_{0}(\mathbf{r}), k_{F}(\mathbf{r})$, and $v_{F}(\mathbf{r})$ are the local equilibrium density, Fermi momentum, and velocity. However, no experimental results are available to compare the theoretical predictions with.

The purpose of the present paper is to compare the performance of various nonadiabatic theories in computing the linewidth of electronic intersubband transitions in single and double square quantum wells-systems in which highquality experimental data exist. ${ }^{17-23}$ Our goal is to clarify the limits of validity of the different approaches, i.e., the ones by Gross and $\mathrm{Kohn}^{4}$ and by VUC (the latter is equivalent to the method by Dobson et al. ${ }^{11}$ in the situation considered here). 
For instance, the formalism of VUC is expected to be exact in the limit of slowly varying density (see above), and in particular does satisfy the HPT. However, this does not necessarily mean that it is the best method under all conditions: it will turn out in this paper that the VUC method works well only for those situations in which the electronic motion is sufficiently collective. If, on the other hand, a single-particlelike behavior prevails (characterized by large gradients in the velocity field), then the method of Gross and $\mathrm{Kohn}^{4}$ gives results which appear to be in better agreement with experiments.

The paper is organized as follows. In Sec. II we introduce the single and double quantum wells used in our comparative studies and calculate their electronic ground state. Section III contains a summary of the linear-response formalism and the various approximations for the linearized xc potential. In Sec. IV we then calculate the lowest collective intersubband transitions for the single and double quantum wells and compare the results to experiment. Conclusions are given in Sec. V.

\section{ELECTRONIC GROUND STATE OF SINGLE AND DOUBLE SQUARE QUANTUM WELLS}

Before discussing the linear response in quantum wells, we need to find their electronic ground state. This problem has been dealt with by several authors before, ${ }^{13,15,19,22,26}$ and we give here a summary of the standard procedure to keep this paper self-contained.

We work in the constant effective-mass approximation, taking a value of $m^{*}=0.07 \mathrm{~m}$ for the effective electron mass in $\mathrm{Ga}_{1-x} \mathrm{Al}_{x} \mathrm{As}$. We furthermore employ a value of 13.0 for the dielectric constant $\epsilon$, so that the electronic charge becomes $e^{*}=e / \sqrt{\epsilon}$. In the following, we choose units such that $e^{*}=m^{*}=\hbar=1$. This means that lengths are measured in units of $1 a_{0}^{*}=98.3 \AA$, and energies in units of 1 hartree $e^{*}=e^{* 2} / a_{0}^{*}=11.27 \mathrm{meV}$. The difference between the lower conduction-band edges of $\mathrm{GaAs}$ and $\mathrm{Al}_{0.3} \mathrm{Ga}_{0.7} \mathrm{As}$ will be taken as $250 \mathrm{meV}$.

We assume that the quantum wells have been grown along the $z$ direction and are translationally invariant in the $x-y$ plane. The Kohn-Sham eigenfunctions for an area $A$ are then discrete in the $z$ direction and plane-wave-like (with wave vector $\mathbf{q}_{\|}$) perpendicular to it. They can be written as

$$
\psi_{\mathbf{q}_{\|}, j}(\mathbf{r})=\frac{1}{\sqrt{A}} e^{i \mathbf{q}_{\|} \cdot \mathbf{r}_{\|}} \varphi_{j}(z),
$$

with the Kohn-Sham eigenvalue

$$
E\left(q_{\|}, j\right)=\frac{q_{\|}^{2}}{2}+\varepsilon_{j} .
$$

The orbitals $\varphi_{j}(z)$ satisfy the following Kohn-Sham equation:

$$
\left(-\frac{1}{2} \frac{d^{2}}{d z^{2}}+v_{\mathrm{ext}}(z)+v_{\mathrm{H}}(z)+v_{\mathrm{xc}}(z)\right) \varphi_{j}(z)=\varepsilon_{j} \varphi_{j}(z) .
$$

Here, the external potential $v_{\text {ext }}(z)$ is prescribed by the design of the quantum well. For the xc potential $v_{\mathrm{xc}}(z)$ we use the LDA of Vosko, Wilk, and Nusair. ${ }^{27}$ The Hartree potential is obtained by direct integration of Poisson's equation $\mathrm{as}^{22}$

$$
v_{\mathrm{H}}(z)=-4 \pi \int_{-\infty}^{z} d z^{\prime} \int_{-\infty}^{z^{\prime}} d z^{\prime \prime} n_{0}\left(z^{\prime \prime}\right)+2 \pi N_{s} z
$$

The sheet density $N_{s}$ is assumed to be a given constant (typically around $10^{11} \mathrm{~cm}^{-2}$ ). To determine the density profile in the $z$ direction, $n_{0}(z)$, we write

$$
\begin{aligned}
n_{0}(z) & =2 \sum_{q_{\|}, j} \varphi_{j}^{2}(z) \theta\left[\varepsilon_{F}-E\left(q_{\|}, j\right)\right] \\
& =\frac{1}{2 \pi} \sum_{\substack{j \\
\varepsilon_{j}<\varepsilon_{F}}} \varphi_{j}^{2}(z)\left(q_{F}^{2}-2 \varepsilon_{j}\right),
\end{aligned}
$$
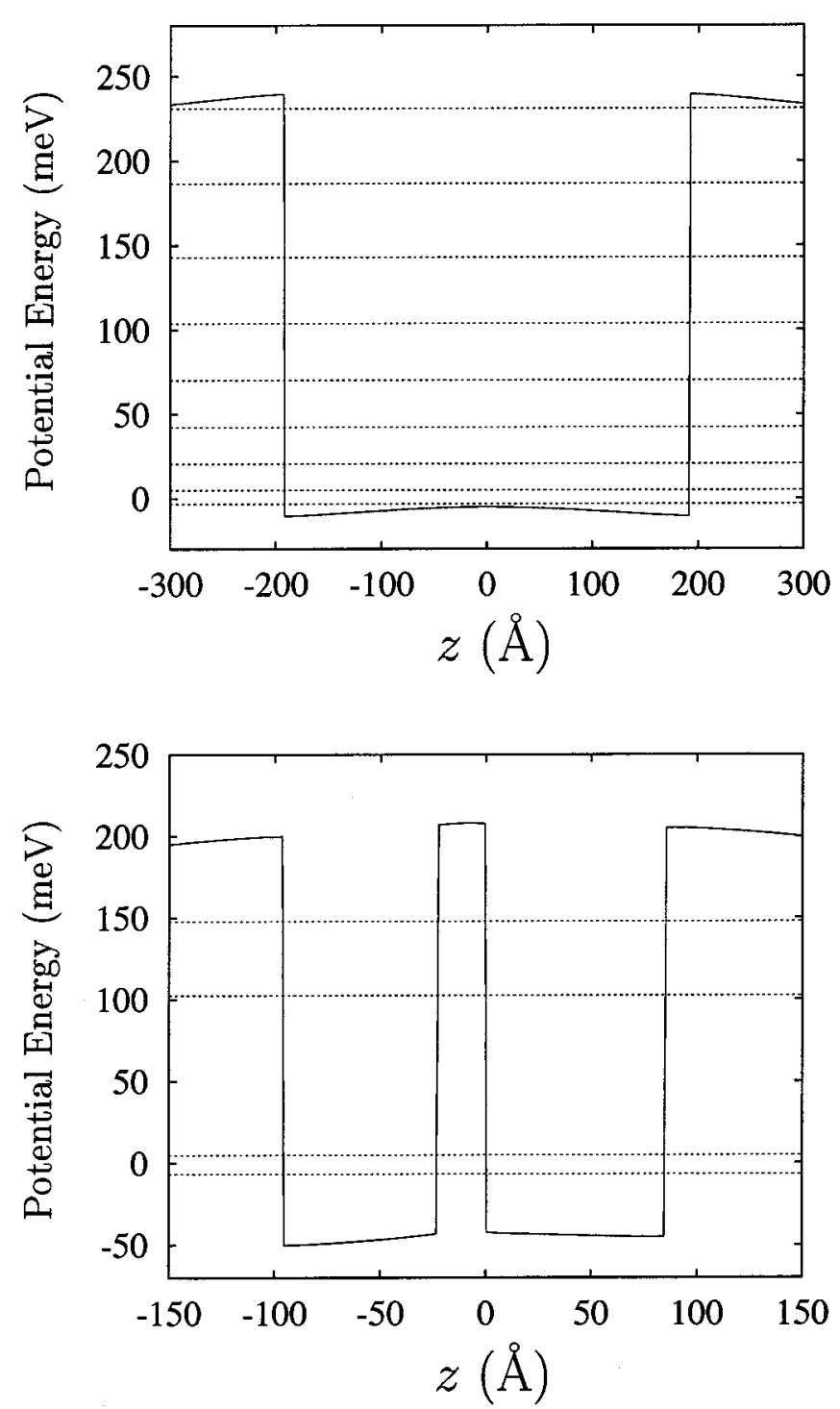

FIG. 1. Full lines: effective Kohn-Sham potential along the growth direction for the electronic ground state of the single and double square wells. The electronic sheet densities are 0.97 $\times 10^{11} \mathrm{~cm}^{-2}$ and $2 \times 10^{11} \mathrm{~cm}^{-2}$, respectively. The dashed lines indicate the bound single-particle levels. The energy scale is such that the Fermi energy lies at zero. Only the lowest level is occupied in both cases. The collective transitions we are interested in take place between the two lowest subbands. 
where $\varepsilon_{F}$ and $q_{F}$ are the Fermi energy and momentum. The sheet density may thus be expressed as

$$
N_{s}=\int d z n_{0}(z)=\frac{1}{2 \pi}\left(q_{F}^{2} N_{\mathrm{occ}}-2 \sum_{j}^{N_{\mathrm{occ}}} \varepsilon_{j}\right),
$$

where $N_{\text {occ }}$ indicates the number of occupied orbitals, and the orbitals themselves are assumed to be normalized to one. To close the self-consistency cycle, we now have to determine $N_{\text {occ }}$. To do this, we solve Eq. (6) for $q_{F}^{2}$ :

$$
q_{F}^{2}=\frac{2 \pi}{N_{\mathrm{occ}}} N_{s}+\frac{2}{N_{\mathrm{occ}}} \sum_{j}^{N_{\mathrm{occ}}} \varepsilon_{j} .
$$

We now start with $N_{\text {occ }}=1$ and, if necessary, keep increasing $N_{\text {occ }}$ until the condition

$$
\varepsilon_{N_{\text {occ }}}<\frac{q_{F}^{2}}{2}<\varepsilon_{N_{\text {occ }}+1}
$$

is satisfied. In the cases we are interested in (see below), only the lowest subband is occupied, i.e., $N_{\text {occ }}=1$.

Let us now discuss specific examples of a single and a double square quantum well, both of which have been experimentally studied. The single GaAs square well ${ }^{17,18}$ is taken to have a width of $384 \AA$. Note that this value is still within the $\pm 4 \%$ range around the design width $400 \AA$ of the sample used in the experiments. ${ }^{18}$ The well is filled with electrons up to a sheet density of $N_{s}=0.97 \times 10^{11} \mathrm{~cm}^{-2}$. In the following, we consider only the case of zero gate voltage and neglect any built-in static electric fields.

The (asymmetric) double quantum well ${ }^{19-23}$ consists of two GaAs wells of widths $85 \AA$ and $73 \AA$, separated by a
$23 \AA$ barrier of $\mathrm{Al}_{03} \mathrm{Ga}_{07}$ As. Its electronic sheet density is $N_{s}=2 \times 10^{11} \mathrm{~cm}^{-2}$. Again, we consider only the case of zero electric field across the sample.

We have solved the Kohn-Sham equation (3) for the two systems within the LDA. For our choices of $N_{s}$, in both cases only the lowest level is occupied in the ground state. The distribution of the bound levels is illustrated in Fig. 1, which also indicates the total effective Kohn-Sham potential of the systems. The energy scale is chosen such that the Fermi level lies at zero. We see that the single square well supports nine bound levels whose spacing grows with increasing energy. The lowest subband spacing is obtained as $E_{12}=8.18 \mathrm{meV}$. The double well, on the other hand, is found to have only four bound levels. Here, the subband spacings are $E_{12}=11.7 \mathrm{meV}, E_{13}=109.2 \mathrm{meV}$, and $E_{14}$ $=154.5 \mathrm{meV}$.

In the following, we take the ground state of these systems and search for the lowest collective intersubband transition using linear-response theory.

\section{LINEAR-RESPONSE FORMALISM FOR QUANTUM WELLS}

\section{A. Response equation}

The linear density response within TDDFT is given by

$$
n_{1}(\mathbf{r}, \omega)=\int d^{3} r^{\prime} \chi_{\mathrm{KS}}\left(\mathbf{r}, \mathbf{r}^{\prime}, \omega\right) v_{\mathrm{eff}}\left(\mathbf{r}^{\prime}, \omega\right),
$$

where $\chi_{\mathrm{KS}}\left(\mathbf{r}, \mathbf{r}^{\prime}, \omega\right)$ is the noninteracting density-density response function, which in our case reads

$$
\chi_{\mathrm{KS}}\left(\mathbf{r}, \mathbf{r}^{\prime}, \omega\right)=2 \sum_{\substack{\mu \nu \\ \mathbf{k}_{\|}, \mathbf{k}_{\|}^{\prime}}}^{\infty}\left(f_{\mu}-f_{\nu}\right) \frac{\psi_{\mathbf{k}_{\|}, \mu}(\mathbf{r}) \psi_{\mathbf{k}_{\|}^{\prime}, \nu}^{*}(\mathbf{r}) \psi_{\mathbf{k}_{\|}, \mu}^{*}\left(\mathbf{r}^{\prime}\right) \psi_{\mathbf{k}_{\|}^{\prime}, \nu}\left(\mathbf{r}^{\prime}\right)}{\omega-\left[E\left(k_{\|}, \mu\right)-E\left(k_{\|}^{\prime}, \nu\right)\right]+i \eta},
$$

with the usual Fermi occupation factors $f_{\mu}$ and $f_{\nu}$, and

$$
v_{\mathrm{eff}}(\mathbf{r}, \omega)=v_{\mathrm{ext}, 1}(\mathbf{r}, \omega)+v_{\mathrm{H}, 1}(\mathbf{r}, \omega)+v_{\mathrm{xc}, 1}(\mathbf{r}, \omega) .
$$

Here, $v_{\text {ext, } 1}(\mathbf{r}, \omega)$ is the frequency-dependent external perturbation, and $v_{\mathrm{H}, 1}(\mathbf{r}, \omega)$ and $v_{\mathrm{xc}, 1}(\mathbf{r}, \omega)$ are the linearized Hartree and xc potential. Since our quantum wells are translationally invariant in the $x-y$ plane, we define

$$
n_{1}\left(\mathbf{q}_{\|}, z, \omega\right)=\int d^{2} r_{\|} e^{-i \mathbf{q}_{\|} \cdot \mathbf{r}_{\|}} n_{1}(\mathbf{r}, \omega),
$$

so that the response equation (9) may be transformed into

$$
n_{1}\left(\mathbf{q}_{\|}, z, \omega\right)=\int d z^{\prime} \chi_{\mathrm{KS}}\left(\mathbf{q}_{\|}, z, z^{\prime}, \omega\right) v_{\mathrm{eff}}\left(\mathbf{q}_{\|}, z^{\prime}, \omega\right) .
$$

In the following, we shall only be concerned with the case in which $q_{\|}=0$ in Eq. (13). The linearized Hartree potential is then

$$
v_{\mathrm{H}, 1}(z, \omega)=-4 \pi \int_{-\infty}^{z} d z^{\prime} \int_{-\infty}^{z^{\prime}} d z^{\prime \prime} n_{1}\left(z^{\prime \prime}, \omega\right)
$$

The first-order xc potential will be discussed below.

For the Kohn-Sham response function, we follow Refs. 26 and 28 and obtain

$$
\begin{aligned}
\chi_{\mathrm{KS}}\left(\mathbf{q}_{\|}, z, z^{\prime}, \omega\right)= & \sum_{\mu=1}^{N_{\mathrm{occ}}} \sum_{\nu=1}^{\infty} F_{\mu \nu}\left(q_{\|}, \omega\right) \varphi_{\mu}(z) \varphi_{\mu}\left(z^{\prime}\right) \\
& \times \varphi_{\nu}(z) \varphi_{\nu}\left(z^{\prime}\right),
\end{aligned}
$$

with the definition 


$$
\begin{aligned}
F_{\mu \nu}\left(q_{\|}, \omega\right)= & \frac{-2}{(2 \pi)^{2}} \int d^{2} k_{\|}\left\{\frac{f\left(\varepsilon_{\mu}+k_{\|}^{2} / 2\right)}{\mathbf{q}_{\|} \mathbf{k}_{\|}+a_{\mu \nu}\left(q_{\|}\right)+\omega+i \eta}\right. \\
& \left.+\frac{f\left(\varepsilon_{\mu}+k_{\|}^{2} / 2\right)}{\mathbf{q}_{\|} \mathbf{k}_{\|}+a_{\mu \nu}\left(q_{\|}\right)-\omega-i \eta}\right\}
\end{aligned}
$$

and

$$
a_{\mu \nu}\left(q_{\|}\right)=\frac{q_{\|}^{2}}{2}-\left(\varepsilon_{\mu}-\varepsilon_{\nu}\right) .
$$

Setting $q_{\|}=0$ in Eqs. (16) and (17) and performing the integration over $k_{\|}$, we end up with

$$
\begin{aligned}
F_{\mu \nu}\left(q_{\|}=0, \omega\right)= & -\frac{\varepsilon_{F}-\varepsilon_{\mu}}{\pi}\left\{\frac{1}{\varepsilon_{\nu}-\varepsilon_{\mu}+\omega+i \eta}\right. \\
& \left.+\frac{1}{\varepsilon_{\nu}-\varepsilon_{\mu}-\omega-i \eta}\right\} .
\end{aligned}
$$

This, together with Eq. (15), defines the response function $\chi_{\mathrm{KS}}\left(\mathbf{q}_{\|}=0, z, z^{\prime}, \omega\right)$. In the following, we will evaluate $\chi_{\mathrm{KS}}$ summing only over the bound states $\varphi_{\nu}$, i.e., the ones shown in Fig. 1. This approximation is justified by the fact that the collective excitations we are studying are well below the continuum threshold for the quantum wells under consideration and thus involve only the lowest-lying states.

\section{B. Approximations for the xc potential}

In our case, the general expression for $v_{\mathrm{xc}, 1}$ depends only on the $z$ coordinate, and is given by

$$
v_{\mathrm{xc}, 1}(z, \omega)=\int d z^{\prime} f_{\mathrm{xc}}\left(z, z^{\prime}, \omega\right) n_{1}\left(z^{\prime}, \omega\right),
$$

where the xc kernel $f_{\mathrm{xc}}\left(z, z^{\prime}, \omega\right)$ is a functional of the ground-state density $n_{0}(z)$.

The simplest possible approximation for $v_{\mathrm{xc}, 1}$ is the ALDA, which assumes

$$
f_{\mathrm{xc}}^{\mathrm{ALDA}}\left(z, z^{\prime}, \omega\right)=\left.\delta\left(z-z^{\prime}\right) \frac{d^{2} e_{\mathrm{xc}}^{\mathrm{hom}}(n)}{d n^{2}}\right|_{n=n_{0}(z)},
$$

where $e_{\mathrm{xc}}^{\mathrm{hom}}(n)$ is the xc energy density of the homogeneous electron gas, so that

$$
v_{\mathrm{xc}, 1}^{\mathrm{ALDA}}(z, \omega)=\left.n_{1}(z, \omega) \frac{d^{2} e_{\mathrm{xc}}^{\mathrm{hom}}(n)}{d n^{2}}\right|_{n=n_{0}(z)} .
$$

In other words, the xc kernel in the ALDA has no frequency dependence at all and is purely real. This approximation is justified for cases in which the external potential is slowly varying in time as well as in space.

In a first step towards overcoming the restriction to slow variation in time, Gross and $\mathrm{Kohn}^{4}$ proposed the following plausible approximation (which we denote by DLDA, the D standing for "dynamic"), valid for the case where both $n_{0}$ and $n_{1}$ are slowly varying in space:

$$
v_{\mathrm{xc}, 1}^{\mathrm{DLDA}}(z, \omega)=f_{\mathrm{xc}}^{\mathrm{hom}}\left(n_{0}(z), q=0, \omega\right) n_{1}(z, \omega),
$$

where $f_{\mathrm{xc}}^{\text {hom }}$ is a property of the homogeneous electron gas, related to the dynamical local field factor as $f_{\mathrm{xc}}^{\text {hom }}\left(n_{0}, \omega\right)$ $=-\lim _{q \rightarrow 0} 4 \pi G(q, \omega) / q^{2}$. Gross et al. ${ }^{4}$ have given a simple parametrization of $f_{\mathrm{xc}}^{\text {hom }}\left(n_{0}, \omega\right)$ (see also Ref. 2). A more elaborate calculation of $f_{\mathrm{xc}}^{\text {hom }}\left(n_{0}, \omega\right)$ has recently been performed by Nifosi, Conti, and Tosi ${ }^{29,30}$ using an approximate decoupling scheme of the equation of motion for the current density, which accounts for processes of excitation of two electron-hole pairs.

As already discussed in the Introduction, the DLDA (22) has recently been found to violate the HPT (for more details, see Refs. 8-10). However, a local frequency-dependent approximation which satisfies the HPT can be derived if the current density is taken as the basic variable, rather than the particle density. The resulting first-order xc vector potential in the form stated by VUC (Ref. 10) is given by

$$
\begin{aligned}
i \omega a_{\mathrm{xc}, 1, \mu}^{\mathrm{VUC}}(\mathbf{r}, \omega)= & \nabla_{\mu} v_{\mathrm{xc}, 1}^{\mathrm{ALDA}}(\mathbf{r}, \omega) \\
& -\frac{1}{n_{0}(\mathbf{r})} \sum_{\nu} \boldsymbol{\nabla}_{\nu} \sigma_{\mathrm{xc}, \mu \nu}(\mathbf{r}, \omega) .
\end{aligned}
$$

The dynamical correction to the ALDA xc potential is the divergence of the viscoelastic stress tensor

$$
\sigma_{\mathrm{xc}, \mu \nu}=\eta_{\mathrm{xc}}\left(\nabla_{\nu} u_{1, \mu}+\nabla_{\mu} u_{1, \nu}-\frac{2}{3} \boldsymbol{\nabla} \cdot \mathbf{u}_{1} \delta_{\mu \nu}\right)+\zeta_{\mathrm{xc}} \boldsymbol{\nabla} \cdot \mathbf{u}_{1} \delta_{\mu \nu} .
$$

Here, $\mathbf{u}_{1}(\mathbf{r}, \omega)=\mathbf{j}_{1}(\mathbf{r}, \omega) / n_{0}(\mathbf{r})$ is the velocity field, and $\eta_{\mathrm{xc}}\left(\omega, n_{0}(\mathbf{r})\right)$ and $\zeta_{\mathrm{xc}}\left(\omega, n_{0}(\mathbf{r})\right)$ are complex viscosity coefficients whose explicit form is given in Ref. 10.

In the one-dimensional case, all quantities depend only on $z$, and current and velocity have $z$ components only, denoted by $j_{1}(z, \omega)$ and $u_{1}(z, \omega)$. The stress tensor then becomes diagonal, and the only element that survives in Eq. (23) is

$$
\sigma_{\mathrm{xc}, z z}=\left(\zeta_{\mathrm{xc}}+\frac{4}{3} \eta_{\mathrm{xc}}\right) \frac{\partial u_{1}}{\partial z}
$$

The combination $\zeta_{\mathrm{xc}}+\frac{4}{3} \eta_{\mathrm{xc}}$ can be expressed as ${ }^{10}$

$$
\begin{aligned}
\zeta_{\mathrm{xc}}+\frac{4}{3} \eta_{\mathrm{xc}} & =-\frac{n_{0}^{2}}{i \omega}\left[f_{\mathrm{xc}}^{\mathrm{hom}}\left(n_{0}, \omega\right)-\left.\frac{d^{2} e_{\mathrm{xc}}^{\mathrm{hom}}(n)}{d n^{2}}\right|_{n=n_{0}}\right] \\
& \equiv-\frac{n_{0}^{2}}{i \omega} f_{\mathrm{xc}}^{\mathrm{dyn}}(z, \omega)
\end{aligned}
$$

Via the relation

$$
v_{\mathrm{xc}, 1}^{\mathrm{VUC}}(z, \omega)=-i \omega \int_{z}^{\infty} d z^{\prime} a_{\mathrm{xc}, 1, \mathrm{z}}^{\mathrm{VUC}}\left(z^{\prime}, \omega\right)
$$

it is then straightforward to convert the $\mathrm{xc}$ vector potential into the corresponding xc scalar potential. Using the linearized continuity equation in one dimension,

$$
i \omega n_{1}=\frac{\partial j_{1}}{\partial z}=\frac{\partial}{\partial z}\left(n_{0} u_{1}\right)
$$

we arrive at ${ }^{9}$ 


$$
\begin{aligned}
v_{\mathrm{xc}, 1}^{\mathrm{VUC}}(z, \omega)= & f_{\mathrm{xc}}^{\mathrm{hom}}\left(n_{0}(z), \omega\right) n_{1}(z, \omega)-\frac{n_{0}^{\prime}(z)}{n_{0}(z)} f_{\mathrm{xc}}^{\mathrm{dyn}}(z, \omega) \int_{-\infty}^{z} d z^{\prime \prime} n_{1}\left(z^{\prime \prime}, \omega\right)-\int_{z}^{\infty} d z^{\prime} \frac{n_{0}^{\prime}\left(z^{\prime}\right)}{n_{0}\left(z^{\prime}\right)} f_{\mathrm{xc}}^{\mathrm{dyn}}\left(z^{\prime}, \omega\right) n_{1}\left(z^{\prime}, \omega\right) \\
& +\int_{z}^{\infty} d z^{\prime}\left(\frac{n_{0}^{\prime}\left(z^{\prime}\right)}{n_{0}\left(z^{\prime}\right)}\right)^{2} f_{\mathrm{xc}}^{\mathrm{dyn}}\left(z^{\prime}, \omega\right) \int_{-\infty}^{z^{\prime}} d z^{\prime \prime} n_{1}\left(z^{\prime \prime}, \omega\right)
\end{aligned}
$$

The first term on the right-hand side of Eq. (29) is the DLDA (22). The other terms are needed to satisfy both the HPT as well as Onsager's reciprocity theorem, i.e., symmetry under interchange of $z$ and $z^{\prime}$ of the associated xc kernel $f_{\mathrm{xc}}\left(z, z^{\prime}, \omega\right)$ (see Ref. 9).

Both the DLDA and the VUC approach lead to a complex $\mathrm{xc}$ potential. This means that the frequency of collective modes will turn out to be complex as well, with a negative imaginary part accounting for the damping. As a consequence, care has to be taken to perform the proper analytic continuation of the Kohn-Sham response function and of $f_{\mathrm{xc}}$, both of which are analytic functions in the upper half complex plane, into the lower half complex plane. ${ }^{24,31,32}$

The analytic continuation of the response function (15) with Eq. (18) is straightforward. This is because there are no branch cuts in the frequency region we consider, and the mode frequency will lie sufficiently far away from the poles in $F_{\mu \nu}\left(q_{\|}=0, \omega\right)$. The latter is a many-body effect known as depolarization shift: due to electron-electron interaction, the mode frequency gets shifted away from the energy separating the single-particle levels. Thus, $\chi_{\mathrm{KS}}\left(q_{\|}=0, z, z^{\prime}, \omega\right)$ is analytic across the real axis in the frequency region of interest.

For the analytic continuation of $f_{\mathrm{xc}}$, we proceed as shown Ref. 32. The idea is the following: let us assume we have a parametrization of the imaginary part of $f_{\mathrm{xc}}(\omega)$ on the real frequency axis. Denote this parametrization by $A_{\mathrm{xc}}(\nu)$, where $\nu$ is real. The real part of $f_{\mathrm{xc}}(\omega)$ on the real frequency axis may then be obtained via the standard Kramers-Kronig relations, due to causality. On the other hand, $f_{\mathrm{xc}}(\omega)$ in the upper complex $\omega$ plane may be written as ${ }^{32}$

$$
f_{\mathrm{xc}}^{\mathrm{up}}(\omega)=f_{\infty}+\frac{1}{\pi} \int_{-\infty}^{\infty} \frac{A_{\mathrm{xc}}(\nu)}{\nu-\omega} d \nu, \quad \operatorname{Im} \omega>0,
$$

since it is analytic and bounded in this region. Here, $f_{\infty}$ is the large-frequency limit of $f_{\mathrm{xc}}$. The analytic continuation into the lower complex $\omega$ plane is given by

$$
f_{\mathrm{xc}}^{\mathrm{lo}}(\omega)=f_{\infty}+\frac{1}{\pi} \int_{-\infty}^{\infty} \frac{A_{\mathrm{xc}}(\nu)}{\nu-\omega} d \nu+2 i A_{\mathrm{xc}}(\omega), \quad \operatorname{Im} \omega<0
$$

This expression arises from a continuous deformation of an integration contour, as outlined in Ref. 32. Note that the third term on the right-hand side implies that $A_{\mathrm{xc}}$, originally defined for real frequencies, is now treated as a complex function, to be evaluated at the complex frequency $\omega, \operatorname{Im} \omega<0$. Therefore, in moving down from the real $\omega$ axis into the lower half complex plane, one has to make sure along the way that no branch cuts or poles of $A_{\text {xc }}$ come across. Due to its simple analytic structure, this is always the case for the parametrization of Gross et al. ${ }^{4}$ The expression of Nifosi, Conti, and Tosi, ${ }^{29,30}$ however, has to be handled with some caution for large values of $|\operatorname{Im} \omega|$.

\section{A simplified approach for the linewidth of collective modes}

The linewidth of collective electronic modes can be obtained in a simplified manner. ${ }^{10,25}$ The idea is to first calculate the modes using the ALDA. Outside the regime where Landau damping occurs, the modes will come out undamped, and the mode frequency $\Omega$ and the density profile $n_{1}(z, \Omega)$ are purely real. The damping due to dynamical xc effects is then added on perturbatively. For the onedimensional case, the resulting linewidth (twice the imaginary part of the mode frequency) is given by

$$
\Gamma_{p}=\frac{\left|\operatorname{Re} \int d z u_{1}^{*}(z, \Omega) \frac{\partial}{\partial z} \sigma_{\mathrm{xc}, z z}(z, \Omega)\right|}{\int d z n_{0}(z)\left|u_{1}(z, \Omega)\right|^{2}} .
$$

Here, $u_{1}$ is a purely imaginary quantity via the continuity equation (28). Thus, using expressions (25) and (26) for $\sigma_{\mathrm{xc}, z z}$, we find

$$
\operatorname{Re}\left(u_{1}^{*} \frac{\partial}{\partial z} \sigma_{\mathrm{xc}, z z}\right)=-u_{1}^{*} \frac{\partial}{\partial z}\left(\frac{n_{0}^{2}}{\Omega} \frac{\partial u_{1}}{\partial z} \operatorname{Im} f_{\mathrm{xc}}^{\mathrm{hom}}\right) .
$$

Partial integration then yields

$$
\Gamma_{p}^{\mathrm{VUC}}=\frac{\int d z n_{0}^{2}(z)\left|\frac{\partial u_{1}}{\partial z}\right|^{2}\left|\operatorname{Im} f_{\mathrm{xc}}^{\mathrm{hom}}\left(n_{0}, \Omega\right)\right|}{\Omega \int d z n_{0}(z)\left|u_{1}(z, \Omega)\right|^{2}} .
$$

To obtain the linewidth within the DLDA, it is convenient to rewrite Eq. (23), using relation (27), as

$$
\frac{\partial}{\partial z} \sigma_{\mathrm{xc}, z z}=n_{0} \frac{\partial v_{\mathrm{xc}, 1}^{\mathrm{ALDA}}}{\partial z}-n_{0} \frac{\partial v_{\mathrm{xc}, 1}^{\mathrm{VUC}}}{\partial z}
$$

We then replace $v_{\mathrm{xc}, 1}^{\mathrm{VUC}}$ with $v_{\mathrm{xc}, 1}^{\mathrm{DLDA}}$, see Eq. (22), thus defining $\sigma_{\mathrm{xc}, z z}^{\mathrm{DLDA}}$, and we find

$$
\operatorname{Re}\left(u_{1}^{*} \frac{\partial}{\partial z} \sigma_{\mathrm{xc}, z z}^{\mathrm{DLDA}}\right)=u_{1}^{*} n_{0} \frac{\partial}{\partial z}\left(n_{1} \operatorname{Im} f_{\mathrm{xc}}^{\mathrm{hom}}\right) .
$$

Performing again a partial integration and using the continuity equation (28) yields the DLDA result

$$
\Gamma_{p}^{\mathrm{DLDA}}=\frac{\int d z\left|\frac{\partial j_{1}}{\partial z}\right|^{2}\left|\operatorname{Im} f_{\mathrm{xc}}^{\mathrm{hom}}\left(n_{0}, \Omega\right)\right|}{\Omega \int d z n_{0}(z)\left|u_{1}(z, \Omega)\right|^{2}} .
$$


We see that $\Gamma_{p}^{\text {DLDA }}$ depends on the gradient of the current density $j_{1}$, whereas $\Gamma_{p}^{\mathrm{VUC}}$ depends on the gradient of the velocity field $u_{1}$.

\section{RESULTS AND DISCUSSION}

\section{A. Numerical results}

Let us now apply the linear-response formalism outlined above to the quantum wells whose electronic ground states we have calculated in Sec. II. The goal is to study the collective intersubband transitions between the first two subbands. Our main interest lies in the damping of these modes.

The standard procedure is to set the external potential $v_{\text {ext, } 1}$ in the response equation (13) equal to zero. The resulting integral equation for $n_{1}(z, \omega)$ can then be viewed as an eigenvalue equation, where the complex integral operator acting on $n_{1}(z, \omega)$ has the eigenvalue 1 . In practice, the collective modes are found iteratively after spatial discretization along the $z$ axis, using a standard numerical eigenvalue solver. The real part of the mode frequency, $\Omega=\operatorname{Re} \omega$, then indicates the position of the mode, and its lifetime is given by $\tau=\Gamma^{-1}$, where $\Gamma=2 \operatorname{Im} \omega$.

Alternatively, one may consider the photoabsorption cross section

$$
\sigma(\omega)=\frac{4 \pi \omega}{c} \operatorname{Im} \alpha(\omega)
$$

where $\omega$ is real, and

$$
\alpha(\omega)=-\frac{2}{E} \int d z z n_{1}(z, \omega)
$$

is the dipole polarizability associated with an external potential of the form $v_{\text {ext }, 1}(z, t)=E z \cos (\omega t)$. We expect to find for our quantum wells that $\sigma(\omega)$ is peaked at the frequency of the collective intersubband transition $\Omega$, with a Lorentzian profile of FWHM $\Gamma$.

We have performed both kinds of calculations for the single and double quantum well. In addition, we compare with the linewidths $\Gamma_{p}$ following from the simplified approach presented in Sec. III C. Results obtained with the various approaches and approximations are summarized in Tables I and II. The first columns give the mode frequency $\Omega$ obtained from ALDA calculations for the collective modes. In both cases, $\Omega$ is already quite close to the experimental result shown in the last column (within $4.2 \%$ for the single and within $4.9 \%$ for the double square well). However, as mentioned before, the ALDA does not include damping of the modes. This means that $\sigma(\omega)$ would consist of a $\delta$ peak at $\Omega$.

The second and third columns in Tables I and II give results for $\Omega$ and $\Gamma$ obtained within the DLDA, using the parametrizations of Gross/Kohn and Nifosi/Conti/Tosi for $f_{\mathrm{xc}}^{\mathrm{hom}}$, respectively. Results within the VUC approximation are given in columns four and five, again for the two different parametrizations for $f_{\mathrm{xc}}^{\text {hom }}$. The first and third lines show $\Omega$ and $\Gamma$ as obtained from a solution of the response equation for the lowest eigenmode. In the second and fourth line, we show the position and width of the associated peak in the
TABLE I. Lowest collective intersubband transition of the single $\mathrm{GaAs} / \mathrm{Al}_{x} \mathrm{Ga}_{1-x} \mathrm{As}$ square well, calculated with various approaches for the linearized xc potential. The superscript 1 (2) stands for the parametrization by Gross/Kohn (Nifosi/Conti/Tosi) for $f_{\mathrm{xc}}^{\text {hom }}$. $\Omega$ is the frequency of the mode, $\Gamma$ is its width. The subscripts indicate the computational scheme: search for the eigenmode of the system as a pole in the complex $\omega$ plane $(m)$, calculation of the photoabsorption cross section $(\sigma)$, and using the simplified approach from Sec. III C $(p)$. All numbers are given in meV.

\begin{tabular}{lcccccc}
\hline \hline & ALDA & DLDA $^{1}$ & DLDA $^{2}$ & VUC $^{1}$ & VUC $^{2}$ & Exp. \\
\hline$\Omega_{m}$ & 10.25 & 10.63 & 10.23 & 10.31 & 10.24 & 10.7 \\
$\Omega_{\sigma}$ & & 10.63 & 10.24 & 10.31 & 10.24 & \\
$\Gamma_{m}$ & & 0.683 & 0.655 & 0.128 & 0.104 & 0.53 \\
$\Gamma_{\sigma}$ & & 0.683 & 0.639 & 0.128 & 0.104 & \\
$\Gamma_{p}$ & & 0.686 & 0.636 & 0.130 & 0.104 & \\
\hline \hline
\end{tabular}

photoabsorption cross section $\sigma(\omega)$. Finally, the last line gives the linewidths $\Gamma_{p}$ as obtained from the formulas presented in Sec. III C.

Let us first consider results for the single quantum well; see Table I. We note that for each choice of the xc potential there is an excellent agreement between the results for $\Omega$ and $\Gamma$ computed with the three different methods. The results for $\Gamma_{p}$ are extremely close to the linewidths obtained using the full calculation for the collective mode or the photoabsorption cross section, which demonstrates the reliability of the linewidth formulas (34) and (37).

We find that the Gross/Kohn parametrization for $f_{\mathrm{xc}}^{\text {hom }}$ shifts the mode frequency $\Omega$ from its value in ALDA closer to the experimental value. The agreement is fairly good within the DLDA, but the improvement is only small if the VUC approximation is used. The Nifosi/Conti/Tosi parametrization, on the other hand, leaves the mode frequency $\Omega$ practically unchanged, within DLDA as well as VUC.

Compared to the experimental value of $0.53 \mathrm{meV}$, the linewidth $\Gamma$ comes out about $25 \%$ too high within DLDA, with only small differences between the two parametrizations for $f_{\mathrm{xc}}^{\text {hom }}(0.683$ and $0.655 \mathrm{meV}$, respectively). By contrast, the VUC linewidth is roughly a factor of 5 smaller than the experimental linewidth. It thus seems as if the DLDA yields a better description of the damping of the modes in the square well.

One has to emphasize, however, that the experimental linewidth contains not only damping effects due to electronelectron interaction, but also caused by additional mechanisms such as finite temperature, scattering off impurities, and fluctuations of the width of the well. ${ }^{33,34}$ Therefore, our calculations should provide a strictly lower limit to the mea-

TABLE II. Same as Table I, for the double square well.

\begin{tabular}{lcccrrr}
\hline \hline & ALDA & DLDA $^{1}$ & DLDA $^{2}$ & VUC $^{1}$ & VUC $^{2}$ & Exp. \\
\hline$\Omega_{m}$ & 13.85 & 14.24 & 13.88 & 20.64 & 12.55 & 14.6 \\
$\Omega_{\sigma}$ & & 14.24 & 13.89 & 20.23 & 12.89 & \\
$\Gamma_{m}$ & & 1.00 & 0.403 & 8.55 & 4.15 & 1.17 \\
$\Gamma_{\sigma}$ & & 1.00 & 0.401 & 8.52 & 3.52 & \\
$\Gamma_{p}$ & & 0.988 & 0.406 & 11.07 & 6.50 & \\
\hline \hline
\end{tabular}




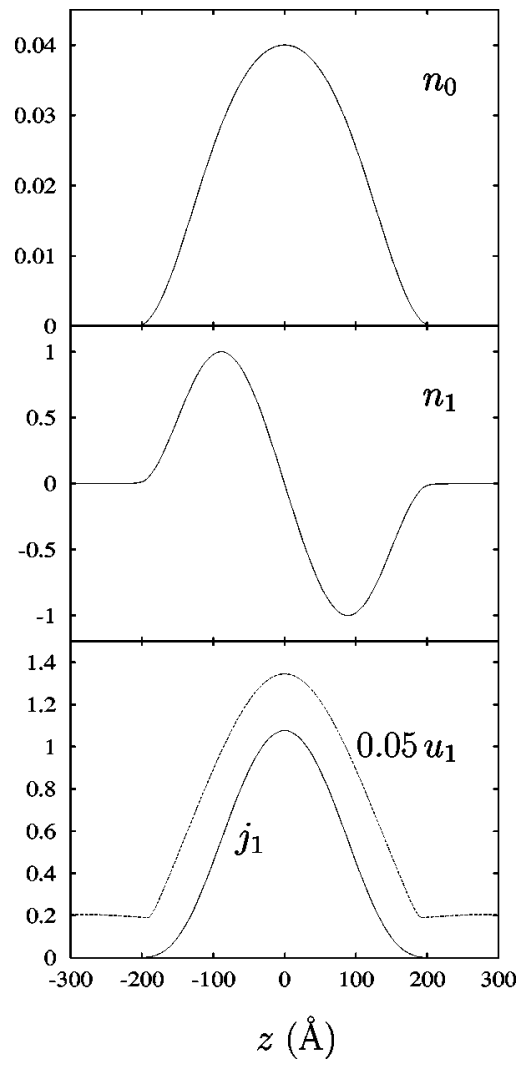

FIG. 2. Top: ground-state density profile $n_{0}(z)$ for the single square well, see Fig. 1 . Middle: density profile $n_{1}(z)$ of the lowest collective mode, calculated within the ALDA. Bottom: associated current density $j_{1}(z)$ and velocity profile $u_{1}(z)$. The latter has been scaled with a factor of 0.05 .

sured linewidth. Clearly, this is the case if the VUC approach is used, whereas, under this aspect, the overestimation of the electronic xc damping within the DLDA is substantial. Note that this effect would be even more drastic in a parabolic well: in that case, VUC (which satisfies the HPT) would correctly give zero damping, whereas DLDA would result in an unphysical finite linewidth.

For the double quantum well, however, the situation is different. From Table II, we see that the DLDA results are at least of similar, sometimes even better quality than for the single well. Again, the mode frequency $\Omega$ agrees better with experiment if the Gross/Kohn parametrization for $f_{\mathrm{xc}}^{\text {hom }}$ is used. In that case, the linewidth is slightly smaller than in the experiment $(1.00 \mathrm{meV}$ compared to $1.17 \mathrm{meV})$. With the Nifosi/Conti/Tosi parametrization, it becomes even smaller by almost a factor of $3(0.403 \mathrm{meV})$.

The VUC method, on the other hand, clearly fails to provide an adequate description for the double quantum well. Within the Gross/Kohn parametrization, the mode frequency $\Omega$ drastically overshoots the experimental value by about $40 \%$. The opposite happens with the Nifosi/Conti/Tosi parametrization, which shifts the ALDA value for $\Omega$ down by more than $1 \mathrm{meV}$. In both cases, the linewidth is dramatically too high. We also observe some differences between the results obtained from calculating the complex mode frequency and the photoabsorption cross section. Moreover, the linewidth formulas (34) and (37) seem to break down here. This obvious failure of the VUC method for the double well

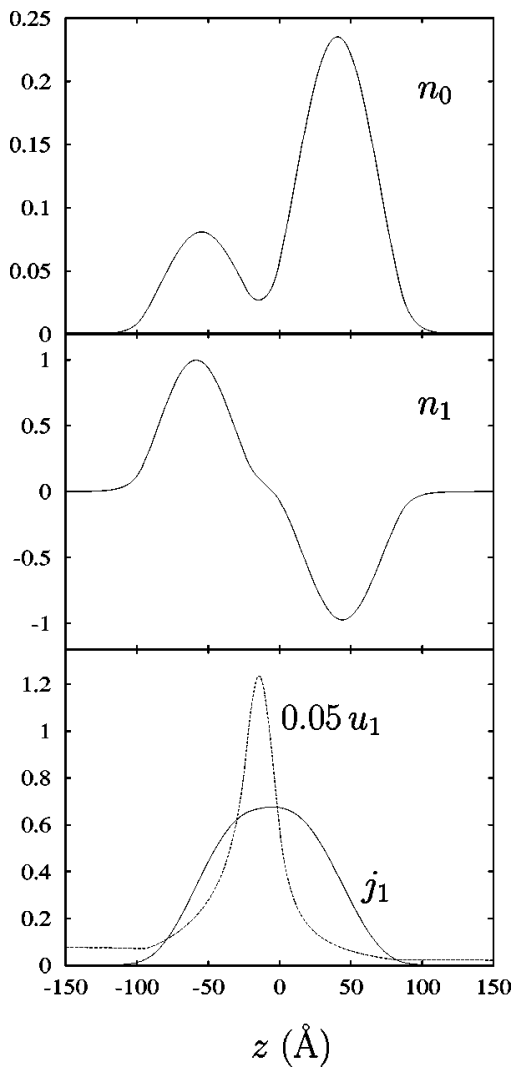

FIG. 3. Same as Fig. 2, for the double quantum well.

calls for some explanation. We shall therefore examine the nature of the electron dynamics in the two quantum wells more closely.

\section{B. Physical picture: Collective versus single particle}

To get some feeling for the underlying physics of the dynamical processes involved, it is helpful to look directly at the local behavior of various quantities characterizing the response of the electron liquid.

Figure 2 shows the ground-state density $n_{0}(z)$ for the single square well and the density profile of its lowest collective mode, $n_{1}(z)$, together with the current density $j_{1}(z)$ and the velocity field $u_{1}(z)$ (here and in the following, we consider the absolute values of $j_{1}$ and $u_{1}$ ). Calculations have been done within the ALDA. The other approximations for the xc potential give very similar results. All quantities plotted in Fig. 2 vary quite smoothly across the well. Note that for the case of a parabolic well, $n_{0}(z)$ and $j_{1}(z)$ would have an identical shape, so that the velocity field $u_{1}(z)$ would be uniform. Here, however, $u_{1}(z)$ has a shape similar to (though slightly smoother than) that of $n_{0}(z)$ and $j_{1}(z)$, which may be viewed as an illustration for the nonparabolicity of the square well.

The same quantities have been plotted for the double quantum well in Fig. 3. Here, the ground-state density $n_{0}(z)$ has two peaks, the lower one at the narrower well and the higher one at the wider well. The dip in $n_{0}(z)$ is at the position of the barrier between the two wells. The density response $n_{1}(z)$ does not deviate too much from the one found for the single well, see Fig. 2, apart from a slight hump at the barrier. The strongest differences, however, show up in 


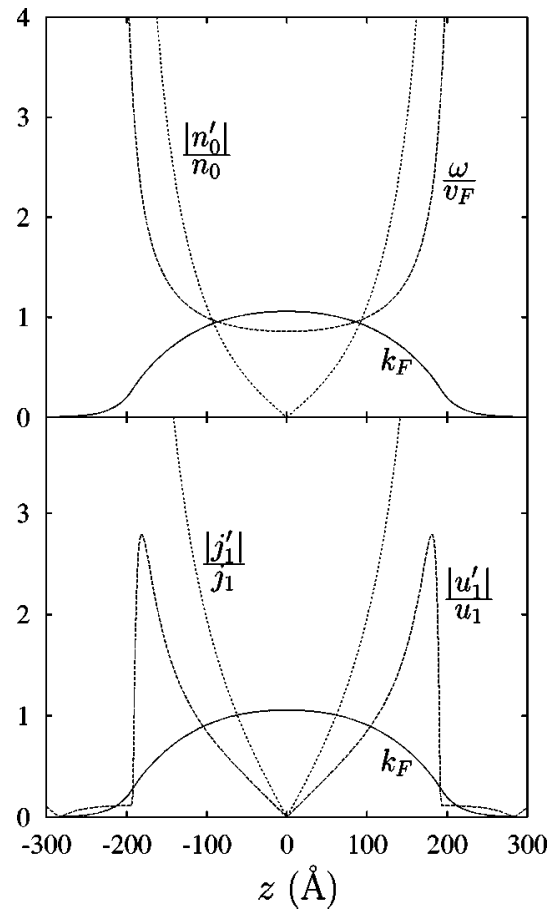

FIG. 4. Top: rate of variation of the ground-state density, $\left|n_{0}^{\prime}(z)\right| / n_{0}(z)$, of the single square well, versus local $k_{F}$ and $\omega / v_{F}$. Bottom: rate of variation of associated current density $j_{1}$ and velocity profile $u_{1}$.

the current density and the velocity field. We see from Fig. 3 that $j_{1}(z)$ is very smoothly varying across the system, apparently ignoring the presence of the barrier between the wells. The velocity field $u_{1}(z)$, however, is strongly peaked at the position of the barrier.

From the examples shown in Figs. 2 and 3, we see that the most sensitive indicator for the physical nature, or rather the degree of "collectiveness," of the motion of an electron liquid is the velocity field $u_{1}(z)$. In general, the electron dynamics in an arbitrary external confining potential will fall somewhere in between two limiting cases: (i) Perfect collective motion. Here we have in mind the example of a parabolic well, i.e., the case upon which the HPT is based. The motion of the center of mass decouples from the relative motion, and the electron liquid as a whole may move rigidly against the positive background ("sloshing mode"). In these cases of no or only little internal compression, the velocity field is uniform or at most only slowly varying on a microscopic scale. (ii) Single-particle-like motion. Here we are thinking of a tunneling process through a classically forbidden region, which is a single-electron phenomenon. Such a process with a large degree of relative motion involves strong internal compression of the electron gas. The presence of a tunneling barrier will locally destroy the coherence of the electronic motion (while the dynamics of the electron gas sufficiently far away from the barrier can still be largely collective). This kind of process is characterized by a pronounced peak in the velocity field, or, more generally speaking, by the presence of large gradients in $u_{1}(z)$. We are thus led to consider more closely the gradients of the various quantities involved.

Note that the domain of validity for the VUC method is restricted by the conditions ${ }^{8-10} \quad k \ll \omega / v_{F}, k_{F}$ and $q$

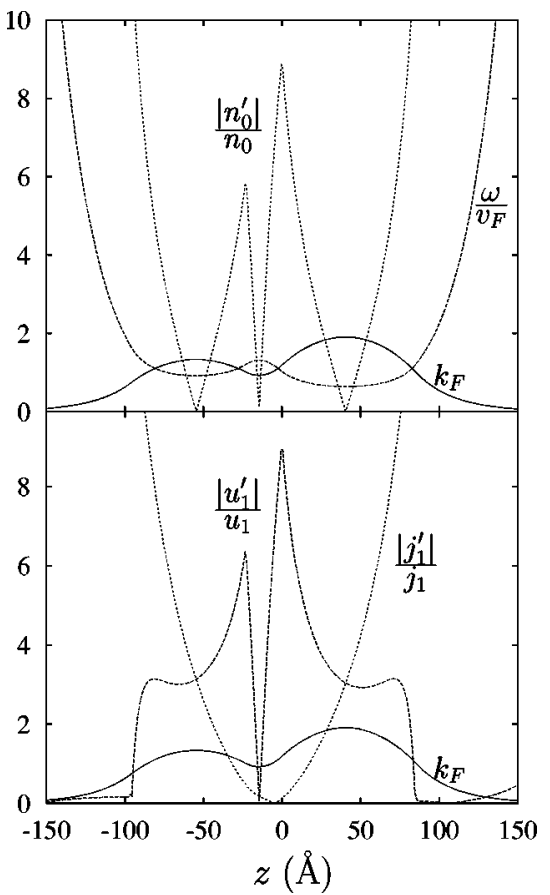

FIG. 5. Same as Fig. 4, for the double quantum well.

$\ll \omega / v_{F}, k_{F}$, where $k^{-1}$ and $q^{-1}$ are the characteristic length scales for variation of the external potential and equilibrium density, respectively, and $k_{F}$ and $v_{F}$ are the local Fermi momentum and velocity. For the problems considered here (calculation of the photoabsorption cross section), the variation of the external potential is negligible, i.e., the condition on $k$ is fulfilled. In turn, a measure for $q$ is given by the quantity $\left|n_{0}^{\prime}(z)\right| / n_{0}(z)$.

The top parts of Figs. 4 and 5 compare the local values of $k_{F}$ and $\omega / v_{F}$ with $\left|n_{0}^{\prime}\right| / n_{0}$ for the single and double well. For the single well, the condition $\left|n_{0}^{\prime}\right| / n_{0}<\omega / v_{F}, k_{F}$ is reasonably well satisfied for the central part of the density distribution, but not towards the borders of the potential well. For the double well in Fig. 5, by contrast, the condition on $\left|n_{0}^{\prime}\right| / n_{0}$ is much more strongly violated-in particular in the central part of the density distribution-due to the fact that there are large density gradients around the region of the potential barrier in the middle.

From the definition of the velocity [see Eq. (28)], we can write

$$
\frac{n_{0}^{\prime}(z)}{n_{0}(z)}=\frac{j_{1}^{\prime}(z)}{j_{1}(z)}-\frac{u_{1}^{\prime}(z)}{u_{1}(z)} .
$$

The two quantities on the right-hand side are plotted in the lower parts of Figs. 4 and 5, again comparing with the local $k_{F}$. We see that for the single quantum well, $\left|u_{1}^{\prime}\right| / u_{1}$ lies consistently below $\left|j_{1}^{\prime}\right| / j_{1}$. For the double well, however, the situation is opposite: $\left|u_{1}^{\prime}\right| / u_{1}$ has sharp structures around the position of the barrier and is much greater than $k_{F}$, whereas $\left|j_{1}^{\prime}\right| / j_{1}$ is relatively smooth over the whole interior of the system. We thus see immediately from Eqs. (34) and (37) that $\Gamma^{\mathrm{VUC}_{\gg}} \Gamma^{\mathrm{DLDA}}$ for the double well is explained by the large values of $\left|u_{1}^{\prime}\right| / u_{1}$ compared to $\left|j_{1}^{\prime}\right| / j_{1}$. Note, finally, 
TABLE III. Same as Table II, for the double square well, using the VUC-DLDA scheme.

\begin{tabular}{lccc}
\hline \hline & VUC-DLDA $^{1}$ & VUC-DLDA $^{2}$ & Exp. \\
\hline$\Omega_{m}$ & 14.07 & 13.87 & 14.6 \\
$\Gamma_{m}$ & 0.620 & 0.194 & 1.17 \\
$\Gamma_{p}$ & 0.605 & 0.192 & \\
\hline \hline
\end{tabular}

that for the case of a parabolic quantum well we would have $n_{0}^{\prime} / n_{0}=j_{1}^{\prime} / j_{1}$ since here the velocity gradient vanishes.

The above analysis suggests that the behavior of the two quantities $\left|u_{1}^{\prime}(z)\right| / u_{1}(z)$ and $\left|j_{1}^{\prime}(z)\right| / j_{1}(z)$ may provide some guidance as to which theoretical approach to choose when $\left|n_{0}^{\prime}(z)\right| / n_{0}(z)$ is not small. We recall that VUC is the correct method in the limit $\left|n_{0}^{\prime}(z)\right| / n_{0}(z) \ll \omega / v_{F}, k_{F}$. If this condition is not satisfied, then, a priori, DLDA and VUC have a comparable status. However, our calculations have shown that when $\left|u_{1}^{\prime}(z)\right| / u_{1}(z)$ is small, then the VUC method is the superior one, whereas when $\left|u_{1}^{\prime}(z)\right| / u_{1}(z)$ is large, then the DLDA works better. This offers a pragmatic way to deal with the question of which method to choose for approximating the dynamic xc potential for an arbitrary system: use VUC only in those regions of space where the velocity field is slowly varying. In those regions where the velocity gradients are large, use the much more robust DLDA. Note that the so-defined VUC-DLDA hybrid scheme automatically satisfies the HPT.

We have applied the VUC-DLDA hybrid scheme to our quantum wells, using VUC where $\left|u_{1}^{\prime}(z)\right| / u_{1}(z)$ $<\left|j_{1}^{\prime}(z)\right| / j_{1}(z)$ and DLDA otherwise. This prescription yields a linewidth which always lies below the linewidth of either of the two "pure" schemes, or at most equals the smaller one of the two. From Fig. 4 it can be seen that for the single well the VUC-DLDA scheme coincides with the VUC method since here the velocity field is always smoother than the current density. In contrast, Fig. 5 for the double well shows that within VUC-DLDA the central part is treated with the DLDA, whereas the VUC is used for the outer region. Numerical results are shown in Table III. Compared to DLDA (see Table II), the resulting mode frequency $\Omega$ is somewhat smaller if the Gross-Kohn parametrization is used, and almost unchanged with the Nifosi/Conti/Tosi parametrization. The effect on the linewidth is more pronounced: for both parametrizations, it is reduced to roughly half of its DLDA value, and is now substantially smaller than the experimental value, as it should be.

Figure 6 illustrates the transition between the "collective" and the "single-particle-like" regime. We calculate the linewidth and lowest collective mode frequency (using ALDA and the linewidth formulas of Sec. III C) for our asymmetric double quantum well with variable barrier height, from its maximum value used so far, down to zero (which then defines a single square well of width $181 \AA$ ). All the while, the electronic sheet density is kept constant. The calculations have been done using the Gross/Kohn parametrization (the other parametrization yields similar results). We see that for decreasing barrier height, the VUC linewidth shrinks much more rapidly than the DLDA linewidth. The crossover occurs around 0.3 times the maximum height. This

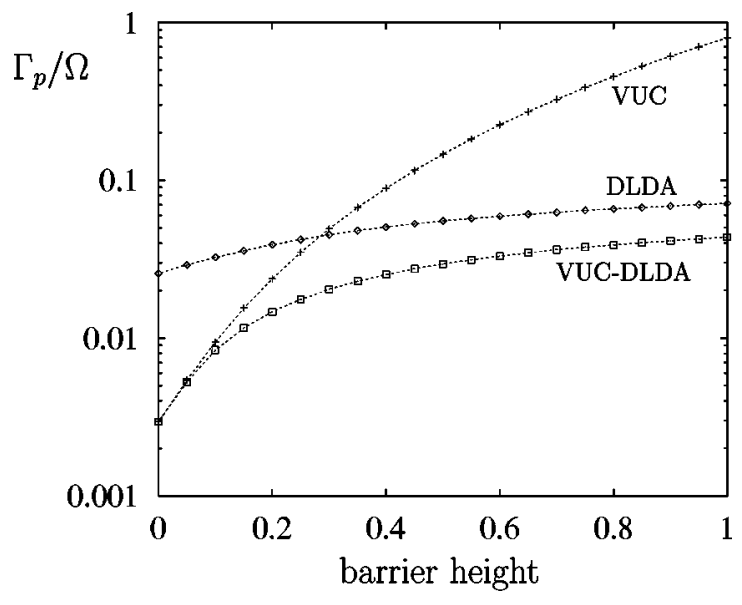

FIG. 6. Ratio of linewidth $\Gamma_{p}$ and lowest collective mode frequency $\Omega$ for the double well with barrier height varying from zero to one, where one corresponds to the full height. Calculations are done with ALDA and the linewidth formulas of Sec. III C, using the VUC, DLDA, and VUC-DLDA hybrid scheme, as indicated.

is the region where the mode frequency becomes comparable to the barrier height. The VUC-DLDA results, on the other hand, lie consistently below the DLDA results, and merge with VUC for small barrier heights, indicating a high degree of collectivity.

\section{CONCLUSION}

In this work, we have carried out a comparative study of two approaches to describe the linear electronic response of quantum wells within a density-functional framework. The two methods are the dynamical extension of the ALDA by Gross and Kohn (DLDA) and the recent implementation of time-dependent current density-functional theory describing dynamical exchange and correlation in the language of conventional hydrodynamics (VUC). The latter approach is rigorously justified in the limit of slowly varying density, in the sense that it satisfies the harmonic potential theorem and other exact Ward identities and symmetries, which in turn are violated by the DLDA. A third approach, recently proposed by Dobson, Bünner, and Gross, ${ }^{11}$ turns out to coincide with VUC in the case of one-dimensional inhomogeneity studied here.

Our studies show that the VUC functional should be applied in general only with a certain caveat, since there are physical situations of interest where it fails to work. Its domain of applicability was found to be closely linked to the structure of the velocity field of the electronic motion. If the latter is sufficiently smooth - which indicates a high degree of collective motion - then VUC leads to sensible results. On the other hand, if there are large gradients of the velocity field (as observed around the nonclassical barrier region in the double well), then VUC is obviously inadequate. By contrast, the DLDA approach was found to be much less sensitive to the nature of the electronic motion and led to a reasonably good description of the double well, although it clearly overdamps the predominantly collective motion in the single well.

We finally proposed a pragmatic answer to the question of 
which functional to choose in a general case. We introduced a hybrid scheme using either VUC or DLDA in their respective spatial region of applicability. This provides a robust method to describe damping of electronic modes of either collective or single-particle-like nature, as shown for the case of a double well with variable barrier height.

\section{ACKNOWLEDGMENTS}

This work was supported by Research Board Grant No. RB 96-071 from the University of Missouri and by NSF Grant No. DMR-9706788. We thank Sergio Conti and Jon Williams for useful discussions.
${ }^{1}$ E. Runge and E. K. U. Gross, Phys. Rev. Lett. 52, 997 (1984).

${ }^{2}$ E. K. U. Gross, J. F. Dobson, and M. Petersilka, in Density Functional Theory II, edited by R. F. Nalewajski, Topics in Current Chemistry Vol. 181 (Springer, Berlin, 1996).

${ }^{3}$ A. Zangwill and P. Soven, Phys. Rev. Lett. 45, 204 (1980); Phys. Rev. B 24, 4121 (1981).

${ }^{4}$ E. K. U. Gross and W. Kohn, Phys. Rev. Lett. 55, 2850 (1985); 57, 923(E) (1986); N. Iwamoto and E. K. U. Gross, Phys. Rev. B 35, 3003 (1987).

${ }^{5}$ J. Dobson, Phys. Rev. Lett. 73, 2244 (1994).

${ }^{6}$ G. Vignale, Phys. Rev. Lett. 74, 3233 (1995).

${ }^{7}$ W. Kohn, Phys. Rev. 123, 1242 (1961); L. Brey, N. F. Johnson, and B. I. Halperin, Phys. Rev. B 40, 10647 (1989); L. Brey, J. Dempsey, N. F. Johnson, and B. I. Halperin, ibid. 42, 1240 (1990); S. K. Yip, ibid. 43, 1707 (1991).

${ }^{8}$ G. Vignale and W. Kohn, Phys. Rev. Lett. 77, 2037 (1996).

${ }^{9}$ G. Vignale and W. Kohn, in Electronic Density Functional Theory, edited by J. F. Dobson, G. Vignale, and M. P. Das (Plenum Press, New York, 1998).

${ }^{10}$ G. Vignale, C. A. Ullrich, and S. Conti, Phys. Rev. Lett. 79, 4878 (1997).

${ }^{11}$ J. F. Dobson, M. J. Bünner, and E. K. U. Gross, Phys. Rev. Lett. 79, 1905 (1997).

${ }^{12}$ P. R. Pinsukanjana, E. G. Gwinn, J. F. Dobson, E. L. Yuh, N. G. Asmar, M. Sundaram, and A. C. Gossard, Phys. Rev. B 46, 7284 (1992).

${ }^{13}$ J. F. Dobson, Phys. Rev. B 46, 10163 (1992).

${ }^{14}$ A. Wixforth, M. Kaloudis, C. Rocke, K. Ensslin, M. Sundaram, J. H. English, and A. C. Gossard, Semicond. Sci. Technol. 9, 215 (1994).

${ }^{15}$ N. G. Asmar and E. G. Gwinn, Phys. Rev. B 46, 4752 (1992).

${ }^{16}$ E. L. Yuh, E. G. Gwinn, P. R. Pinsukanjana, W. L. Schaich, K. L. Campman, P. F. Hopkins, and A. C. Gossard, Phys. Rev. B 54, 11467 (1996).

${ }^{17}$ K. Craig, C. L. Felix, J. N. Heyman, A. G. Markelz, M. S. Sherwin, K. L. Campman, P. F. Hopkins, and A. C. Gossard, Semicond. Sci. Technol. 9, 627 (1994).

${ }^{18}$ K. Craig, Ph.D. thesis, University of California, Santa Barbara, 1997 (unpublished).
${ }^{19}$ J. N. Heyman, K. Craig, B. Galdrikian, M. S. Sherwin, K. Campman, P. F. Hopkins, S. Fafard, and A. C. Gossard, Phys. Rev. Lett. 72, 2183 (1994).

${ }^{20}$ J. N. Heyman, K. Unterrainer, K. Craig, B. Galdrikian, M. S. Sherwin, K. Campman, P. F. Hopkins, and A. C. Gossard, Phys. Rev. Lett. 74, 2682 (1995).

${ }^{21}$ M. S. Sherwin, K. Craig, B. Galdrikian, J. Heyman, A. Markelz, K. Campman, S. Fafard, P. F. Hopkins, and A. Gossard, Physica D 83, 229 (1995).

${ }^{22}$ B. Galdrikian and B. Birnir, Phys. Rev. Lett. 76, 3308 (1996).

${ }^{23}$ J. B. Williams, K. Craig, M. S. Sherwin, K. Campman, and A. C. Gossard, Physica E 2, 177 (1998).

${ }^{24}$ D. Pines and P. Nozières, The Theory of Quantum Liquids (W. A. Benjamin, New York, 1966).

${ }^{25}$ C. A. Ullrich and G. Vignale, Phys. Rev. B 58, 7141 (1998).

${ }^{26}$ J. F. Dobson, in Density Functional Theory, Vol. 337 of NATO Advanced Study Institute Series B: Physics, edited by E. K. U. Gross and R. M. Dreizler (Plenum Press, New York, 1995), p. 393.

${ }^{27}$ R. M. Dreizler and E. K. U. Gross, Density Functional Theory: An Approach to the Quantum Many-Body Problem (SpringerVerlag, Berlin, 1990)

${ }^{28}$ A. G. Eguiluz, Phys. Rev. B 31, 3303 (1985).

${ }^{29}$ H. M. Böhm, S. Conti, and M. P. Tosi, J. Phys.: Condens. Matter 8, 781 (1996); S. Conti, R. Nifosì, and M. P. Tosi, ibid. 9, L475 (1997).

${ }^{30}$ R. Nifosi, S. Conti, and M. P. Tosi, Phys. Rev. B (to be published).

${ }^{31}$ J. F. Dobson and G. H. Harris, J. Phys. C 21, L729 (1988).

${ }^{32}$ J. F. Dobson, G. H. Harris, and A. J. O'Connor, J. Phys.: Condens. Matter 2, 6461 (1990).

${ }^{33}$ R. J. Warburton, K. Weilhammer, J. P. Kotthaus, M. Thomas, and H. Kroemer, Phys. Rev. Lett. 80, 2185 (1998).

${ }^{34}$ To estimate the contribution of the last effect to the line broadening, note that changing the width of the well by one monolayer (about $3 \AA$ ) shifts the real part of the mode frequency by about $0.1 \mathrm{meV}$. 